\title{
Eficacia de tiapride en el mantenimiento de la abstinencia en alcohólicos desintoxicados. Resultados de un ensayo clínico a doble ciego frente a placebo
}

\author{
Gual, A.; Monrás, M.; Ortega, Ll. \\ Unitat d'Alcohologia de la Generalitat. Institut de Psicología i Psiquiatría. Hospital Clínic Mejía Lequerica s/n. 08010 Barcelona. \\ Enviar correspondencia: \\ Antoni Gual i Solé. Unitat d'Alcohologia de la Generalitat. IMD. Hospital Clínic. Villarroel, 170. 08036 Barcelona. \\ Tel. 932275548. Email: tgual@clinic.ub.es
}

\section{RESUMEN}

Fundamentos: Diversos trabajos han descrito la utilidad de Tiapride tanto en la desintoxicación como en la deshabituación alcohólica. Este estudio intenta demostrar su eficacia en la deshabituación de una muestra de alcohólicos españoles.

Material y método: 81 alcohólicos desintoxicados procedentes de 4 Centros distintos y cumpliendo criterios DSM III-R de dependencia, fueron aleatóriamente colocados en el grupo experimental (Tiapride, $\mathrm{n}=38$ ) o en el grupo control (Placebo $\mathrm{n}=43$ ). Todos recibieron Tiapride $100 \mathrm{mg}$ o placebo cada 8 horas y durante de 24 semanas. Se realizaron 11 visitas de control y análisis de sangre las semanas 8,16 y 24. El análisis estadístico se realizó bajo el principio de la intención de tratar considerándose como variables principales el tiempo hasta la primera recaida (TPR) y la duración acumulada de la abstinencia (DAA).

Resultados: Ambos grupos resultaron comparables en cuanto a características sociodemográficas y clínicas. En el grupo placebo las tasas de abandono fueron ligeramente mas altas (18\% versus $35 \%)$ sin llegar a ser significativas $(p<.09)$. A los 6 meses, $49 \%$ pacientes en el grupo placebo y $37 \%$ en el grupo Tiapride permanecian abstinentes, con un TPR de 97 versus 88 dias a favor del grupo placebo $(p<.27)$. Igualmente, la DAA en ambos grupos fue similar (130 dias en el grupo placebo versus 138 en el grupo tiapride; $p<.57)$.

Conclusión: Nuestros resultados no apoyan la hipótesis de que tiapride pueda mejorar las tasas de abstinencia en la fase de deshabituación.

Palabras clave: Alcoholismo, deshabituación, tiapride.

\section{ABSTRACT}

Introduction: Tiapride has been proposed as an adjunct in pharmacological treatment in the postweaning phase of alcoholism treatment, as well as in the detoxification period. This trial attempts to test its efficacy in a sample of alcoholics in Spain.

Material and method: 81 detoxified alcoholics from 4 different centres and with a clinical diagnosis of alcohol dependence (DSM III-R), were randomly allocated to the experimental (tiapride, $n=38$ ) and control (placebo, $n=43$ ) groups. All patients were given tiapride $100 \mathrm{mg}$ (or matching placebo tablets), to be taken every 8 hours for 24 weeks. 11 controls were scheduled and blood samples were collected on weeks 8, 16 and 24. Statistical analysis was performed under the ITT principle, and Time to first Relapse (TFR) and Cumulated Abstinence Duration (CAD) were used as the main outcome measures.

Results: No significant differences were found between both samples in terms of sociodemographic variables, clinical characteristics of alcohol dependence and biological markers of alcoholism. Attrition rates were slightly higher in the placebo group (18\% versus 35\%) without reaching statistical significance ( $p>$.09). At the 6 months follow up, 49\% patients in the placebo group and $37 \%$ in the tiapride group were still abstinent, with a mean TFR of 97 versus 88 days in favour of the placebo group $(p<.27)$. CAD values were also similar for both groups (130 versus 138 days in favour of tiapride; $p<.57$ ).

Conclusion: Our data do not support the hypothesis that tiapride can enhance abstinence rates in weaned alcoholics.

Key words: Alcoholism, treatment, tiapride.

\section{INTRODUCCION}

$\mathbf{E}$ los últimos años el interés de los científicos en el tratamiento farmacológico de la deshabituación alcohólica ha sido creciente. Durante décadas el proceso de deshabituación se ha planteado como un proceso psicoterapéutico donde no tenían cabida los fármacos, (a excepción de los antidipsotrópicos, que además no eran utilizados precisamente como verdaderas medicinas). La aparición de estudios demostrando la eficacia del acamprosato ${ }^{1,2}$, la naltrexona ${ }^{3,4}$ y algunos ISRS ${ }^{5,6}$ en el mantenimiento de la abstinencia o la reducción de los consumos, ha disparado el interés 
de la industria farmacéutica en un campo al que hasta ahora había prestado poca atención.

No todos los estudios arrojan los resultados esperados. Así, merece la pena destacar los resultados negativos obtenidos con algunos fármacos como la bromocriptina ${ }^{7}$ y el flupentixol ${ }^{8}$. Asimismo, en los fármacos donde se ha demostrado un efecto terapéutico existen todavía aspectos del diseño experimental a mejorar ${ }^{9}$, aunque en líneas generales los estudios realizados son metodológicamente correctos ${ }^{10}$.

El tiapride suele clasificarse entre los neurolépticos atípicos. Es un derivado benzamídico con una acción antagonista a nivel de los receptores dopaminérgicos $D_{2}$. Si bien su actividad antidopaminérgica se halla plenamente demostrada, posee también una acción ansiolítica cuyas bases farmacológicas no han sido todavía establecidas con claridad, ya que sólo depende parcialmente de su acción antidopaminérgica ${ }^{11}$.

En el tratamiento del alcoholismo se ha propuesto el uso de fármacos que actúan a nivel del sistema dopaminérgico por múltiples razones. En una excelente revisión Soyka ${ }^{12}$ señala entre otras las siguientes: La ingestión aguda de alcohol produce una liberación de dopamina a nivel del núcleo acumbens, lugar preferente de actuación de las substancias capaces de generar dependencia; en cambio, la ingesta crónica provoca una disminución en la liberación de dopamina y la consiguiente hipersensibilización de los receptores dopaminérgicos. A ello hay que añadir la evidencia existente de la involucración del sistema dopaminérgico en los efectos reforzadores del alcohol; involucración que, al menos parcialmente, viene modulada por el sistema opiáceo endógeno.

A nivel experimental se han publicado numerosos estudios que demuestran la utilidad de tiapride en la desintoxicación alcohólica ${ }^{13,14}$. En cambio, la evidencia de su eficacia en la fase de deshabituación es más escasa. De hecho, un estudio de Delamaire ${ }^{15}$ con 60 pacientes y un seguimiento de sólo 3 semanas encontró diferencias no significativas aunque con una tendencia más favorable para el grupo tratado con tiapride (68 vs 47\% de mejoría). En un trabajo más riguroso realizado por Shaw et $\mathrm{al}^{16}$ con un número reducido de pacientes, sí se hallaron diferencias significativas $(p<.02)$ en la Duración Acumulada de la Abstinencia (103 vs 54 días). Estos resultados fueron replicados por el mismo autor posteriormente ${ }^{17}$. Con una muestra más amplia $(n=100)$ y siempre a 180 días, los resultados fueron nuevamente mejores en el grupo tratado con tiapride (Duración Acumulada de la Abstinencia de 77 vs 49;p<.01).

Aunque de momento no se haya publicado, también es digno de mención un estudio multicéntrico realizado en Alemania (M. Soyka, comunicación personal en el Simposium de la European Society of Biomedical Research on Alcoholism. Barcelona, junio de
1999) en donde tiapride no mostró diferencias significativas frente a placebo.

La disparidad de resultados obtenidos por los distintos investigadores y el creciente interés de los tratamientos farmacológicos en el curso de la deshabituación alcohólica motivaron la realización del presente trabajo, cuyo objetivo es elucidar la eficacia frente a placebo de Tiapride, en una muestra de pacientes alcohólicos españoles.

\section{MATERIAL Y MÉTODO}

Se trata de un estudio prospectivo, multicéntrico, randomizado, a doble ciego, en paralelo, para comparar la eficacia de tiapride frente a placebo en el mantenimiento de la abstinencia alcohólica en pacientes afectos del síndrome de dependencia alcohólica, desintoxicados y en fase de deshabituación. Participaron 4 centros del estado español (Unidad de Alcohología del Hospital Clínic de Barcelona; Servicio de Psiquiatría y Toxicomanías de la Mútua de Terrassa; Servicio de Toxicomanías de Torrente del Hospital General de Valencia; Centro Provincial de Drogodependencias de Málaga), que aportaron un total de 81 pacientes.

Aleatoriamente 38 pacientes fueron asignados a tiapride y 43 a placebo. El tratamiento farmacológico consistió en la toma de tiapride en cápsulas de 100 mg (o placebo en cápsulas de idéntico formato) cada 8 horas y por espacio de seis meses (180 días). En el curso del estudio los pacientes fueron citados para visitas de control semanalmente durante el primer mes, quincenalmente durante los meses segundo y tercero, y cada 4 semanas los 3 últimos meses. El protocolo utilizado incluyó la realización de una historia alcohológica de ítems cerrados, que incorpora la historia toxicológica, datos sociodemográficos y criterios DSM III R de dependencia. Asimismo, en el curso de la visita de inclusión se practicó una analítica general y de funcionalismo hepático, se administraron diversos tests (STAI, BECK), y se realizó determinación de alcohol en orina. Esta última prueba se repitió en todas las visitas de control, como puede comprobarse en la Tabla 1, donde se resumen las pruebas realizadas a lo largo de todo el estudio.

Sólo se incluyeron pacientes que cumpliesen con los siguientes criterios de inclusión: Entre 20 y 65 años de edad, de ambos sexos, que cumpliesen criterios DSM III R de dependencia alcohólica y que se mantuviesen abstinentes de bebidas alcohólicas entre 7 y 15 días en el momento de la inclusión. Se excluyeron aquellos pacientes que estuviesen siguiendo algún tipo de tratamiento psicofarmacológico, aquellos con historia de abuso o dependencia de otras drogas a excepción del tabaco, mujeres gestantes y 


\begin{tabular}{|c|c|c|c|c|c|c|c|c|c|c|c|c|}
\hline & Día & & & & & & & & & & & \\
\hline & 0 & 7 & 14 & 21 & 28 & 42 & 56 & 70 & 84 & 112 & 140 & 168 \\
\hline $\begin{array}{l}\text { Historia clínica } \\
\text { STAI } \\
\text { BECK } \\
\text { VCM } \\
\text { ASAT } \\
\text { ALAT } \\
\text { GGT } \\
\text { Alcohol en orina } \\
\text { Criterios DSM-III-R } \\
\text { Visita médica }\end{array}$ & $\begin{array}{l}x \\
x \\
x \\
x \\
X \\
X \\
x \\
x \\
X \\
x\end{array}$ & $X$ & $x$ & $X$ & $\begin{array}{l}X \\
X \\
X \\
X \\
X \\
X \\
X \\
X \\
X\end{array}$ & $X$ & $x$ & $x$ & $\begin{array}{l}X \\
X \\
X \\
X \\
X \\
X \\
X \\
X \\
X\end{array}$ & $X$ & $\begin{array}{l}x \\
x\end{array}$ & $\begin{array}{l}X \\
X \\
X \\
X \\
X \\
X \\
X \\
X \\
X\end{array}$ \\
\hline
\end{tabular}

pacientes con enfermedades somáticas graves y psicosis.

Se analizaron los resultados bajo el principio de la intención de tratar, considerándose como variable principal de eficacia la duración acumulada de la abstinencia ${ }^{18}$ (DAA). Asimismo, se midió el tiempo hasta la primera recaída (TPR) y la duración de la remisión estable (DRE). La DAA se define como el número de días en que el paciente se ha mantenido abstinente en el curso del ensayo. La DRE mide el tiempo que el paciente lleva abstinente en el momento de finalizar el ensayo, y el TPR mide el tiempo que el paciente se mantuvo abstinente desde el inicio del ensayo. Se consideró que un paciente no se había mantenido abstinente para un determinado período en cualquiera de los siguientes casos: Determinación de alcohol en orina positiva, autoreporte del paciente reconociendo consumos alcohólicos y no acudir a las visitas programadas.

El tiempo hasta la primera recaída y la retención de pacientes en tratamiento se compararon mediante la técnica de Kaplan Meier (Log Rank) para el análisis de supervivencia. Para comparar la duración acumulada de la abstinencia y la duración de la remisión estable en ambas muestras se utilizó el t-test de Student. Los porcentajes de abandonos y consumidores a los 6 meses en ambos grupos se han comparado mediante la prueba de ji cuadrado.

\section{RESULTADOS}

La muestra la constituyen 81 pacientes con una edad promedio de 40 años, predominando los varones $(85 \%)$, casados $(77 \%)$, con un alcoholismo de inicio anterior a los 15 años $(68 \%)$ y un elevado porcentaje con antecedentes familiares de dependencia alcohólica $(50 \%)$. A nivel clínico y sociodemográfico no se encontraron diferencias significativas entre ambos grupos, ni tampoco en cuanto a los valores de los distintos marcadores biológicos y cuestionarios utilizados (Tablas 2 y 3 ).

\begin{tabular}{|c|c|c|c|}
\hline \multicolumn{4}{|c|}{$\begin{array}{c}\text { Tabla 2. Características clínicas y } \\
\text { sociodemográficas de ambos grupos } \\
\text { experimentales al inicio del tratamiento. }\end{array}$} \\
\hline & Tiapride & Placebo & p \\
\hline Edad & $38 \pm 6$ & $41 \pm 10$ & ,063 \\
\hline Sexo (Varones) & $84 \%$ & $86 \%$ & ,816 \\
\hline $\begin{array}{l}\text { Estado Civil } \\
\text { (Casados) }\end{array}$ & $80 \%$ & $75 \%$ & ,878 \\
\hline $\begin{array}{l}\text { Escolaridad } \\
\text { (Primaria) }\end{array}$ & $47 \%$ & $47 \%$ & ,657 \\
\hline $\begin{array}{l}\text { Alcoholismo } \\
\text { familiar (si) }\end{array}$ & $47 \%$ & $52 \%$ & ,654 \\
\hline $\begin{array}{l}\text { Edad de inicio } \\
\text { (<15 años) }\end{array}$ & $69 \%$ & $67 \%$ & ,997 \\
\hline
\end{tabular}

Tabla 3. Valores analíticos y psicométricos basales en ambos grupos experimentales.

\begin{tabular}{|lccc|}
\hline & Tiapride & Placebo & $\mathbf{p}$ \\
\hline VCM & 99 & 104 &, 093 \\
GGT & 188 & 147 &, 289 \\
ASAT & 54 & 57 &, 735 \\
ALAT & 53 & 54 &, 862 \\
STAI-S & 21 & 20 &, 711 \\
STAI-T & 30 & 27 &, 193 \\
BECK & 16 & 15 &, 935 \\
\hline
\end{tabular}

El cumplimiento del tratamiento fue ligeramente mejor en el grupo tiapride, observándose en la curva de supervivencia una tasa mayor de abandonos en el grupo placebo (39\% placebo vs $21 \%$ tiapride; Log 
Rank $=2.49, p=0.115)$ aunque sin significación estadística (Figura 1). Contrariamente a lo esperado, al medir el tiempo hasta la primera recaída las tendencias se invierten, tal como se observa en el Figura 2, siendo el grupo placebo quien obtiene mejores resul-

Figura 1. Atrición en ambos grupos. Análisis de supervivencia.

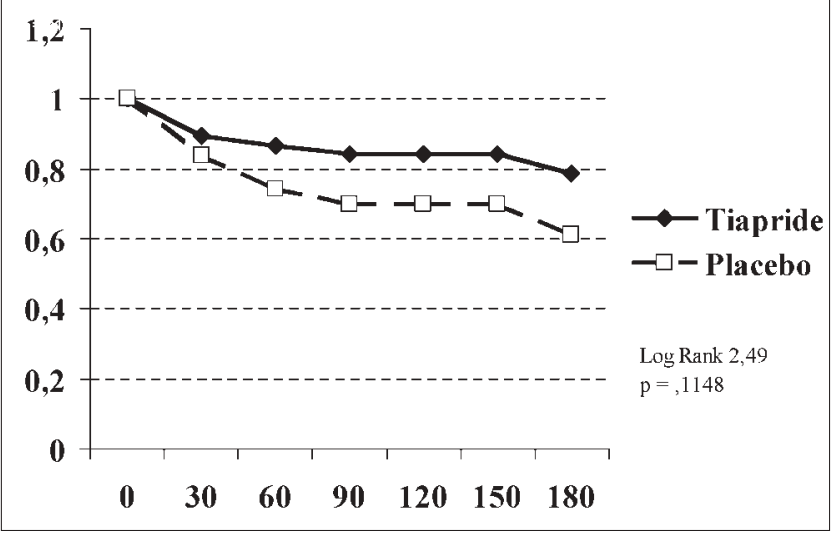

Cuando el análisis de supervivencia se realiza contabilizando el tiempo de seguimiento de los pacientes que abandonan pero sin considerar el abandono como recaída, los resultados se tornan de forma estadísticamente significativa favorables al placebo $169 \%$ frente a 30\% de abstinencia a los 180 días; Log Rank =6.26, $\mathrm{p}<.012$ ). Dado que el análisis principal se realizó bajo el principio de la Intención de tratar, estos datos deben entenderse como un análisis secundario de significación clínica cuestionable (Figura 3).

En el análisis realizado bajo el principio de la intención de tratar y utilizando como variable principal la DAA, las diferencias entre ambos grupos no son significativas $(t=.57 ; p=.57)$, obteniéndose una DAA de 138 días para el grupo tiapride frente a 130 días en el grupo placebo. Al analizar la DRE los resultados vuelven a invertirse (114 días para el grupo placebo frente a 89 en el grupo tiapride) aunque siguen sin ser estadísticamente significativas las diferencias $(t=1.49 ; p=.14)$. Finalmente, si en vez de considerar la evolución de los pacientes como dicotómica (abstinencia y recaída), establecemos tres categorías posibles (abstinencia, consumo y abandono del tratamiento), observamos cómo ambas muestras se comportan de formas distintas y a nivel estadísticamente significativo (ji-cuadrado= $6.2 ; p=.04)$. En efecto, mientras en el grupo tiapride predominan los pacientes que beben y continúan en tratamiento, en el grupo placebo se observan tasas más altas de abstinencia, así como de abandono. En la Tabla 4 se detallan estos resultados.

Finalmente, el análisis de las variables secundarias aporta pocos datos interesantes. No se observan diferencias significativas en cuanto a los valores de los marcadores biológicos del consumo de alcohol. En tados, presentando un TPR de 97 días frente a los 88 días del grupo tiapride. Ello comporta un 44\% de abstinencia a los 180 días frente al 27\% del grupo tiapride, sin que ninguna de estas diferencias sea significativa (Log Rank $=.82 ; p=.366)$.

Figura 2. Tiempo hasta la primera recaída. Análisis de supervivencia considerando los abandonos de tratamiento como fracasos.

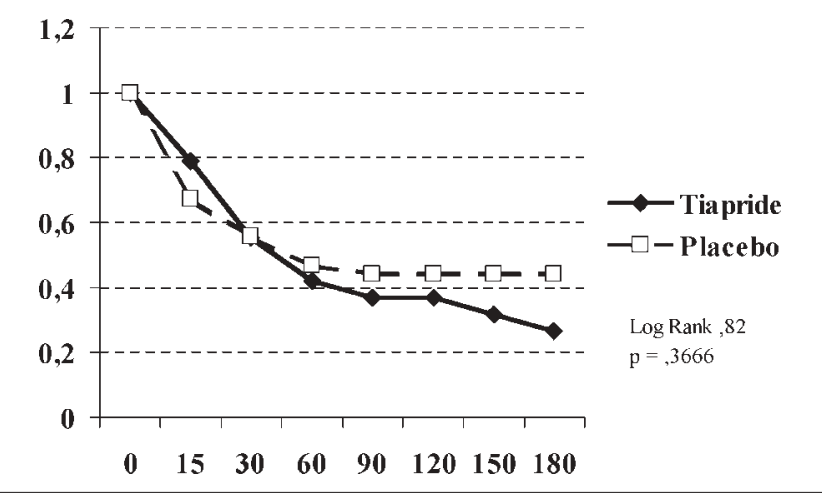

Figura 3. Tiempo hasta la primera recaída. Análisis de supervivencia censurando los pacientes que abandonan el tratamiento.

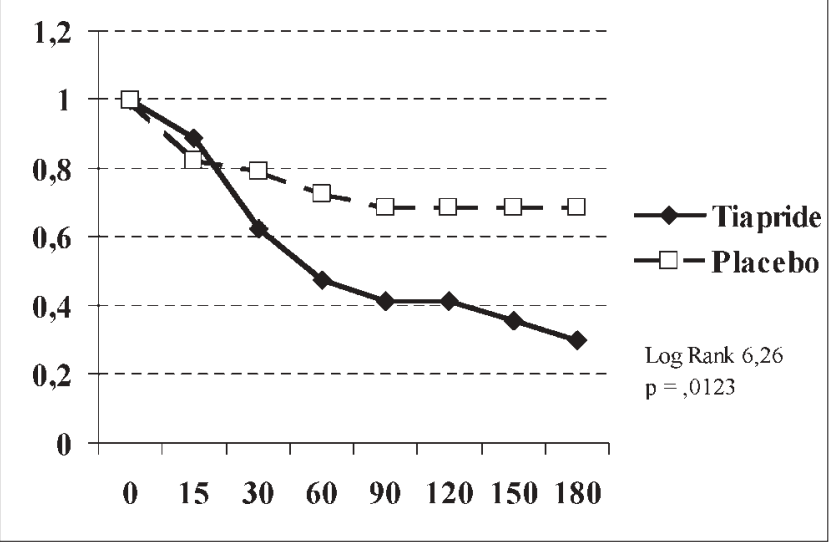

\begin{tabular}{|l|c|c|c|c|c|c|}
\hline \multicolumn{7}{|c|}{ Tabla 4. } \\
Diferencias en la situación clínica de los \\
pacientes al finalizar el ensayo, \\
en función del tipo de tratamiento*.
\end{tabular}


cambio, los valores que miden depresión se mantienen significativamente más elevados en el grupo tiapride que en el placebo, aunque estos datos deben ser valorados con cautela puesto que en ningún caso alcanzan niveles patológicos y además se refieren lógicamente sólo a pacientes que han finalizado el tratamiento (Tabla 5).

Tabla 5. Valores analíticos y psicométricos en ambos grupos al finalizar el tratamiento*.

\begin{tabular}{|l|c|c|c|} 
& Tiapride & Placebo & p \\
\hline STAI - S & $17,3 \pm 11$ & $11,7 \pm 11$ &, 066 \\
BECK & $8,8 \pm 9$ & $4 \pm 2$ &, 01 \\
VCM & $93,7 \pm 5$ & $93,8 \pm 5$ &, 94 \\
GGT & $47,5 \pm 37$ & $31,8 \pm 25$ &, 08 \\
ASAT & $25 \pm 16$ & $27 \pm 12$ &, 63 \\
ALAT & $29,8 \pm 28$ & $33,4 \pm 18$ &, 6 \\
\hline
\end{tabular}

* Se incluyen en el análisis sólo los pacientes que finalizaron el ensayo clínico.

\section{DISCUSIÓN}

Los resultados obtenidos en este estudio no demuestran ninguna diferencia significativa en favor de tiapride frente a placebo que justifique su utilización en la fase de deshabituación alcohólica. El análisis principal realizado bajo el principio de la intención de tratar y considerando los abandonos de tratamiento como recaídas no halla diferencias significativas entre ambos grupos experimentales al medir los consumos alcohólicos en términos de DAA, DRE y tiempo hasta la primera recaída.

Un análisis más pormenorizado de los datos permite ver una desigual distribución de la evolución en ambas muestras. Así, en el grupo tiapride predominan los pacientes que recaen y siguen en tratamiento, mientras que en el grupo placebo son los abstinentes quienes constituyen el grupo más numeroso.

Se produce así una curiosa situación donde los pacientes tratados con principio activo presentan una mayor retención en tratamiento, sin que ello comporte una mejor evolución clínica sino todo lo contrario. De hecho, cuando sólo se analiza los pacientes que han seguido el tratamiento correctamente, los que fueron tratados con placebo obtienen mejores resultados en el tiempo hasta la primera recaída que los pacientes del grupo tiapride.

Este estudio obtiene resultados distintos de los anteriormente publicados. Cabe reseñar aquí que estos resultados son similares a los del estudio multicéntrico alemán, no publicado aún, y reabren una importante reflexión entorno a la dificultad existente para publicar estudios con fármacos cuando los resultados son negativos.

Asimismo, los ensayos publicados con resultados positivos merecen especial atención. El trabajo de Delamaire et al ${ }^{15}$ es cuestionable desde el punto de vista metodológico (asignación no aleatoria a 3 grupos), por el tamaño de la muestra y muy especialmente por su corta duración (3 semanas). Asimismo, sorprende la interpretación positiva de los autores cuando los resultados muestran tendencias que no son estadísticamente significativas. Los trabajos de Shaw ${ }^{16,17}$, aunque ya poseen un mayor rigor metodológico adolecen también de un sesgo importante, puesto que el análisis principal se realiza en los pacientes que terminan el tratamiento, cuando en la actualidad se halla plenamente establecido que los análisis de eficacia deben realizarse bajo el principio de la intención de tratar. En nuestro caso se comprueba como los resultados difieren cuando se analiza el total de la muestra o sólo los pacientes que finalizan el tratamiento (Figuras 2 y 3), aunque precisamente en este último grupo es donde encontramos una mejor respuesta al placebo, contrariamente a lo observado por Shaw.

En resumen pues, el análisis de nuestros resultados no permite recomendar el uso sistemático de tiapride en la deshabituación alcohólica. Ello no excluye que pueda ser de utilidad en algunos pacientes, habiéndose observado una ligera tendencia a mejorar la retención en tratamiento, sin que ello comporte un aumento en las tasas de abstinencia medidas mediante la CAD y elTPR.

\section{AGRADECIMIENTOS}

El trabajo de campo en los distintos centros fue coordinado por Lluisa Ortega, Félix Bravo, Miguel Ángel Torres y Jordi Sanahuja. Este trabajo fue realizado con la financiación de Laboratorios Delagrange S.A., que facilitaron también la medicación y placebo utilizados.

\section{REFERENCIAS BIBLIOGRÁFICAS}

(1) Sass H, Soyka M, Mann K, Zieglgansberger W Relapse prevention by acamprosate. Results from a placebocontrolled study on alcohol dependence. Arch Gen Psychiatry (United States), 1996; 53(8):673-80.

(2) Poldrugo F Acamprosate treatment in a long-term community-based alcohol rehabilitation programme. Addiction (England) 1997; 92(11):1537-46.

(3) O'Malley SS, Jaffe AJ, Chang G, Schottenfeld RS, Meyer RE, Rounsaville B Naltrexone and coping skills therapy 
for alcohol dependence. A controlled study. Arch Gen Psychiatry (United States), 1992; 49(11):881-7.

(4) Volpicelli JR, Alterman Al, Hayashida M, O'Brien CP Naltrexone in the treatment of alcohol dependence Arch Gen Psychiatry (United States), 1992; 49(11):87680.

(5) Pettinati, H. Placebo-controlled trial of sertraline for primary alcohol dependence. Alcohol Alcohol (England), 1999; 34(3): 432.

(6) Angelone SM, Bellini L, Di Bella D, Catalano M Effects of fluvoxamine and citalopram in maintaining abstinence in a sample of Italian detoxified alcoholics. Alcohol Alcohol (England), 1998; 33(2):151-6.

(7) Naranjo CA, Dongier M, Bremner KE Long-acting injectable bromocriptine does not reduce relapse in alcoholics. Addiction (England), 1997; 92(8):969-78.

(8) Boening J, Wiesbeck GA, Weijers HG, Lesch OM, Glaser T, Toennes PJ, Flupenthixol decanoate for relapse prevention in alcohol dependence. Results from an international, double blind multicenter trial. Alcohol Alcohol (England), 1999; 34(3): 455.

(9) Moncrieff J, Drummond DC New drug treatments for alcohol problems: a critical appraisal. Addiction (England), 1997; 92(8):939-47.

(10) Gual, A. Editorial. Alcoscope (England), 1998; Vol 1(3): 1.

(11) Peters DH, Faulds D. Tiapride. A review of its pharmacology and therapeutic potential in the management of alcohol dependence syndrome. Drugs (New Zealand), 1994; 47(6):1010-32.

(12) Soyka, M. Relapse prevention in alcoholism. Recent advances and future possibilities. CNS Drugs (New Zealand), 1997; 7(4): 313-327.

(13) Lepola U, Kokko S, Nuutila J, Gordin A. Tiapride and chlordiazepoxide in acute alcohol withdrawal. A controlled clinical trial. Int J Clin Pharmacol Res (Switzerland), 1984; 5:321-326.

(14) Murphy DJ, Shaw GK, Clarke I. Tiapride and chlormethiazole in alcohol withdrawal: a double blind trial. Alcohol Alcohol (England), 1983; 18: 227-237.

(15) Delamaire D, Carpentier MC, Eudier F, Derouet J, Bourel M Essai ambulatoire du tiapridal aprés sevrage alcoholique hospitalier. Semaine des Hôpitaux de Paris 1986; 62: 3109-3114.

(16) Shaw GK, Majumdar SK, Waller S, MacGarvie J, Dunn G Tiapride in the long term management of alcoholics of anxious or depressive temperament. Br J Psychiatry (England), 1987; 150,: 164-168.

(17) Shaw GK, Waller S, Majumdar SK, Alberts JL, Latham CJ, Dunn G Tiapride in the prevention of relapse in recently detoxified alcoholics. $\mathrm{Br} J$ Psychiatry (England), 1994; 165(4):515-23.

(18) Lehert P Review and discussion of statistical analysis of controlled clinical trials in alcoholism. Alcohol Alcohol (England), 1993; Suppl 2:157-163. 